\title{
Hereditary and Path Coalgebras
}

\author{
William Chin \\ DePaul University, Chicago, Illinois 60614 \\ wchin@condor depaul.edu
}

September 11, 2000

\section{Introduction}

It is a well-known that, over an algebraically closed field and up to Morita equivalence, finite dimensional hereditary algebras are exactly the finite dimensional path algebras (see e.g. [Be]). In this note, we give short proof of the dual of this fundamental fact, which holds for arbitrary coalgebras and path coalgebras of quivers, without finiteness assumptions.

The results of this article were recently obtained differently in [JMLS] where hereditary coalgebras are studied via a notion of formal smoothness. Here we present a short and direct approach.

Background material may be found in $[\mathrm{C}, \mathrm{Mo}, \mathrm{Sw}]$. We let $Q$ be a quiver and let $k Q$ denote the path coalgebra (see [CMo] or [C]) over the field $k$, with comultiplication $\Delta$. For a path $p, s(p)$ denotes the starting vertex. Let $\left\{C_{n}\right\}$ denote the coradical filtration of the coalgebra $C$. By the quiver of $C$, we mean the Ext-quiver as in [C,CMo]. A coalgebra is said to be hereditary [NTZ] if homomorphic images of injective comodules are injective.

Our main result is

Theorem 1 Every pointed hereditary coalgebra is isomorphic to the path coalgebra of its quiver.

Corollary 2 Every hereditary coalgebra over an algebraically closed field is Morita-Takeuchi equivalent to the path coalgebra of its (Ext-) quiver.

To prove these statements we rely on

Lemma 3 Let $C$ be a coalgebra with a hereditary subcoalgebra $D$. If $C_{1} \subset D$, then $C=D$.

Proof. Let $C$ and $D$ be as in the hypothesis. First notice that if $D \wedge C_{0}=D$, then $D=C$ (dual Nakayama Lemma [Sw]). Consequently (since $D \wedge C_{0}$ is a subcoalgebra containing $D$ ) we may assume that $D \wedge C_{0}=C$; i.e.,

$$
\Delta C \subset D \otimes C+D \otimes C_{0}
$$


Therefore $C / C_{0}$ is a left $D$ - comodule. The inclusion $D / D_{0} \hookrightarrow C / C_{0}$ splits since $D / D_{0}$ is injective. Contensoring the inclusion with $C_{0}$ (i.e. taking socles) yields the injection $D_{1} / D_{0} \hookrightarrow C_{1} / C_{0}$. But $C_{1} / C_{0}=D_{1} / D_{0}$ is the socle of $C / C_{0}$. This forces $(C / D)_{0}=0$ and hence $C / D=0$. This completes the proof of the Lemma.

Proof of Theorem and Corollary: Every coalgebra is Morita-Takeuchi equivalent to a basic coalgebra. Over an algebraically closed field basic coalgebras are precisely the pointed ones. Now let $D$ be a hereditary pointed coalgebra. Then by [CMo], $D$ embeds in the path coalgebra $C$ of its quiver so that the copy of $D$ contains $C_{1}$. By the Lemma, the image of $D$ is all of $C$.

Conversely we have:

Theorem 4 Every path coalgebra is hereditary.

Proof. Let $C=k Q$. By [NTZ, Theorem 4] it suffices to show that $C / S$ is injective for simple right coideals $S=k g$, where $g$ is a vertex. Thus, letting $I$ denote an indecomposable injective right coideal containing $S$, it suffices to show that $I / S$ is injective. Here of course $I$ is an injective hull of $S$, and $I$ is isomorphic to the span of paths ending at $g$.

Let $A_{g}$ denote the set of arrows ending at $g$. For each $\alpha \in A_{g}$, let $I(\alpha)$ be an injective hull of $k s(\alpha)$.

For all $\alpha \in A_{g}$,we define a map $f_{\alpha}: I \rightarrow I(\alpha)$ by letting $d_{\alpha} \in \operatorname{Hom}_{k}(C, k)$ be defined by $d_{\alpha}(p)=\delta_{a, p}$, for paths $p$ (Kronecker $\delta$ ), and setting $f_{\alpha}=\left\llcorner d_{\alpha}=\right.$ $\left(d_{\alpha} \otimes 1\right) \Delta$ using the "hit" action, see [Mo]. The map $f_{\alpha}$ is a right comodule homomorphism. The reader can quickly check that $f_{\alpha}$ can be seen to truncate paths ending at with $\alpha$, i.e., $f_{\alpha}(\alpha p)=p, f_{\alpha}(\alpha)=s(\alpha)$ and is zero on paths not ending with $\alpha$.

Now set

$$
f=\oplus_{\alpha \in A_{g}} f_{\alpha}: I \rightarrow \oplus_{\alpha \in A_{g}} I(\alpha) .
$$

Then it is easy to see that $f$ is onto, and that the kernel of $f$ is $\mathrm{kg}$. The image is an injective comodule since the direct sum of injective comodules is injective. This shows that is $I / S$ injective as required.

\section{References}

[Be] D. Benson, Representations and cohomology I. Basic representation theory of finite groups and associative algebras, Second edition. Cambridge Studies in Advanced Math., 30. Cambridge University Press, 1998.

[C] W Chin, a brief introduction to coalgebra representation theory, Conf. Proc. Morelia, Michoacan 1997; E. Green, R. Bautista and R. Martinez, eds. (to appear). 
[CMo] W. Chin and S. Montgomery, Basic Coalgebras, in Modular Interfaces (Riverside, CA, 1995) AMS/IP Studies in Advanced Math. vol.4, Providence RI, (1997) 41-47.

[JMLS] P. Jara, L. Merino, D. Llena, D. Stefan, Formally smooth coalgebras, preprint Granada 2000.

[Mo] Hopf algebras and Their Actions on Rings, CBMS Regional Conference Series in Mathematics, 82, American Mathematical Society, Providence, RI, 1993.

[NTZ] Nastasescu, B. Torrecillas, Y.H. Zhang, Hereditary coalgebras, Comm. Alg. 24(4), 1521-1528 (1996).

[Sw] Sweedler, M., Hopf Algebras, Benjamin, New York 1969. 